\title{
A nanobiosensor to detect single hybridization events $\dagger$
}

\section{Giuseppe Maruccio, ${ }^{*}$ Elisabetta Primiceri, Pasquale Marzo, Valentina Arima, Antonio Della Torre, Ross Rinaldi, Teresa Pellegrino, $\$$ Roman Krahne $\$$ and Roberto Cingolani $\$$}

\author{
Received 1st October 2009, Accepted 14th October 2009 \\ First published as an Advance Article on the web 23rd October 2009 \\ DOI: $10.1039 / b 920559 b$
}

\begin{abstract}
An economical nanoarray method to electrically detect hybridization events is demonstrated. As a proof of concept, we fabricated a sensor for DNA sequencing, in which targets are oligonucleotides conjugated to gold nanoparticles. As a consequence of target-probe binding events, a conductive bridge forms between two electrodes, resulting in a quantized change in conductivity. This enables a robust detection of a few (down to single) hybridization events and can be potentially applied also to other binding events (like specific interactions between proteins, antibodies, ligands and receptors). Moreover, target amplification techniques (such as PCR) are no longer necessary.
\end{abstract}

\section{Introduction}

Microarrays are widely used for DNA sequencing, disease screening, gene expression analysis and proteomics applications since they provide information in a faster and simpler way than traditional methods. ${ }^{1}$ Such devices typically employ optical readouts requiring sophisticated detection equipment and labeling of the molecules with fluorescent markers. In addition, for the detection of nucleic acids, an additional step for sample preparation, such as polymerase chain reaction (PCR) or similar target-amplification techniques, is required to amplify the DNA and reach the detection threshold. Electrical detection methods are therefore desirable to overcome such limitations by virtue of their large-scale integrability, easy read-out and higher sensitivity. Here, we demonstrate a DNA array detection device in which electrical nanojunctions are shorted when targets bind to capture probes. The target is an oligonucleotide conjugated to conductive nanoparticles (e.g. Au nanocrystals (AuNCs)), whereas the probe is an oligonucleotide immobilized on nanoelectrodes. As a consequence of a target-probe binding event, a conductive bridge forms between the electrodes, ${ }^{2}$ resulting in a discrete change of the electrical conductivity with single biorecognition event sensitivity. This enables a robust electrical detection of DNA hybridization events and our method can be potentially extended to any other specific binding events, such as interactions between proteins, antibodies, ligands and receptors. The device layout exploits a recently-demonstrated interconnection technique for the cheap fabrication of large-scale arrays of nanojunctions without complex high-resolution lithography processes, thus opening the way to combinatorial sequence analysis by solid-state chips with electrical read-out.

National Nanotechnology Laboratory of CNR-INFM, Scuola Superiore ISUFI, Università del Salento, IIT-Research Unit, v. Arnesano 16, I-73100 Lecce, Italy.E-mail: giuseppe.maruccio@unisalento.it

$\uparrow$ Electronic supplementary information (ESI) available: additional figures describing the detection strategy, the characterization of AuNC-DNA conjugates by gel electrophoresis and hybridization tests on gold surfaces. See DOI: 10.1039/b920559b

+ Present address: Italian Institute of Technology, Via Morego, 30, I-16163 Genova, Italy.

\section{Nanosensor fabrication}

Our prototype device consists of two main parts: a nanojunction array (the transducer) and the molecular probes, specifically designed for the biorecognition event to be monitored, immobilized on the electrodes. The targets are marked with conductive nanoparticles. The device fabrication consist of three phases

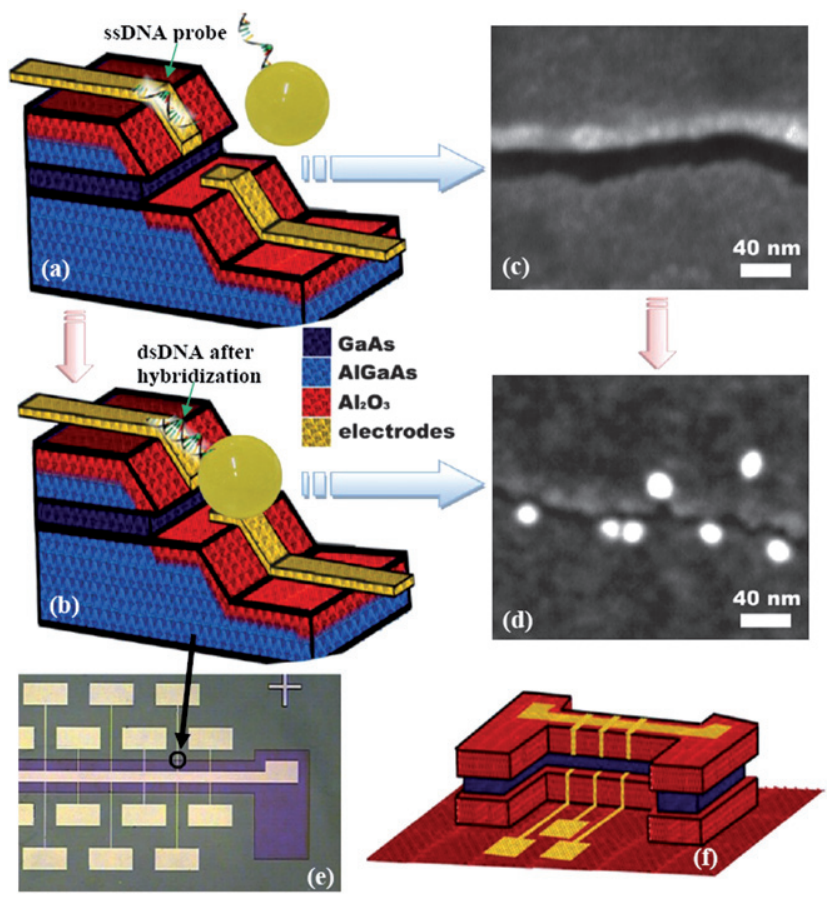

Fig. 1 Nanojunction array and detection scheme. (a-b) Schematics of the mesa nanojunctions with immobilized ssDNA capture probes (a) before and (b) after hybridization with target ssDNA labeled with gold nanoparticles (not to scale). (c) Scanning electron microscopy image of a nanojunction before hybridization. (d) SEM image after hybridization: a number of nanoparticles immobilized on the electrodes and close to the gap are visible. (e-f) Optical micrograph and 3D schematics of a nanojunction array. The lateral size of the electrodes is about $2 \mu \mathrm{m}$, while the gap is about $20 \mathrm{~nm}$. 
(Fig. 1): (i) the conjugation of biomolecules with conductive nanoparticles; (ii) the fabrication of the nanojunction array; and (iii) the immobilization of the capture probes on the electrode surface. As proof of concept, we fabricated a hybridization sensor for DNA sequencing. As capture probes and targets, we used complementary oligonucleotides functionalized with alkanethiols $\left(\mathrm{C}_{6}-\mathrm{SH}\right)$ at their $5^{\prime}$-termini (5'-SH-ssDNA) in order to be immobilized on the electrodes and conjugated to gold nanoparticles, respectively. As conductive markers, we used AuNCs of $20 \mathrm{~nm}$ mean diameter purchased from Ted Pella, Inc. in aqueous solutions. The AuNC-DNA conjugates were prepared by addition of $5^{\prime}$-SH-ssDNA ${ }^{3}$ to the AuNC solution and successively characterized by gel electrophoresis and atomic force microscopy to test the accessibility of oligonucleotides in conjugates and investigate the influence of the ssDNA packing density (see the Methods section).

To detect single biorecognition events, we employed arrays of nanojunctions. Since any approach involving electron beam lithography (EBL) is not suitable for mass production (due to its low throughput and related high cost), non-conventional solutions for creating nanojunctions are needed for massive application to point-of-care analysis. For these reasons, we recently developed a new interconnection scheme ${ }^{4,5}$ for the simultaneous fabrication of large arrays of nanodevices by the selective wet-etching and oxidation of an $\mathrm{AlGaAs} / \mathrm{GaAs}$ quantum well structure (Fig. 1a). In detail, an $\mathrm{AlGaAs} / \mathrm{GaAs} / \mathrm{AlGaAs}$ quantum well structure is grown by molecular beam epitaxy and subsequently carved to a slanted shape by wet-etching, until reaching the bottom AlGaAs layer. Then a few tens of nanometers of the GaAs layer are removed by selective wet-etching with citric acid and $\mathrm{H}_{2} \mathrm{O}_{2}$ and the $\mathrm{AlGaAs}$ barriers are converted in insulating oxide layers by means of a selective oxidation. Finally, the electrode pattern is defined by simple optical lithography and the electrodes are fabricated by metal evaporation. The result (Fig. 1c) is a vertical nanogap which can be controlled with subnanometer precision by the width of the GaAs quantum well and the thickness of the deposited metal layer. This process is easily parallelised in large arrays like that shown in Fig. 1e-f.

To enable detection, 5'-SH-ssDNA capture probes are immobilized on the electrodes (Fig. 1) in a mixed self-assembled monolayer (SAM) with 2-mercaptoethanol molecules as spacers to improve the hybridization efficiency. Specifically, the nanojunctions are first incubated for $2 \mathrm{~h}$ in an aqueous DNA solution diluted at $1 \mu \mathrm{M}$ concentration in a $1 \mathrm{M} \mathrm{KH}_{2} \mathrm{PO}_{4}$ solution, and then for $1 \mathrm{~h}$ in a $1 \mathrm{mM}$ 2-mercaptoethanol solution. To evaluate the sensitivity of the nanosensor array, the functionalized nanojunctions are incubated in a $0.5 \mathrm{nM}$ solution $(12.5 \mathrm{mM}$ phosphate buffer and $25 \mathrm{mM} \mathrm{NaCl}$ ) containing appropriate AuNC-DNA conjugates with the DNA strands complementary to the capture probes on the electrodes. After incubation, the devices are washed in PBS and in a $0.3 \mathrm{M}$ solution of ammonium acetate in order to remove DNA strands unspecifically-bonded.

\section{Results}

As a consequence of a biorecognition event the AuNCs selfassemble onto the nanoelectrodes and bridge the electrode gap, as illustrated in Fig. 1b. High-resolution SEM images of the nanogap area before (Fig. 1c) and after hybridization (Fig. 1d) clearly show that the AuNC-DNA conjugates immobilize on the DNA functionalized electrodes. These hybridization events are detected as a discrete increase in conductivity of the nanojunction. Fig. 2 shows current-voltage characteristics (blue, green and red curves) for three different nanojunctions, where hybridization between complementary ssDNA has occurred. Measurements are performed on dried junctions. We observe a non-linear $I-V$ characteristic with current amplitudes in the nanoAmpere range at a bias voltage of $1 \mathrm{~V}$. As a negative test we also show the (black) curve that corresponds to the empty nanojunction after functionalization with the ssDNA capture probes. In this case, the current at a bias voltage of $1 \mathrm{~V}$ is of the order of some hundred picoAmperes, therefore one order of magnitude lower than in the case of the recognition event. All $I-V$ curves after hybridization exhibit similar sigmoidal shapes, with higher resistances at small voltages, that fit a Simmons model $^{6}$ for tunneling with similar values for the barrier height (around $0.53 \pm 0.01 \mathrm{eV}$ ). By comparing the current amplitude of the different curves at higher bias voltage, for example $1 \mathrm{~V}$, we find that we can assume $N=1,2$ and 3 hybridization events for the red, green and blue curves respectively, since the conductance $G$ appears to increase in steps of a conductance quantum $G^{*}$ corresponding to a single-event/AuNC junction $\left(G^{*} \approx 10 \mathrm{nA} \mathrm{V}^{-1}\right)$. As shown in the inset, in fact, the curves collapse on almost the same characteristics if we divide the current by $N$, as expected for the case of $N$ conductive channels in the gap. The single bioevent sensitivity allows us to avoid amplification of the DNA target, or other signal amplification techniques such as silver enhancement. As a control experiment and to test the selectivity of our sensors, we fabricated and characterized devices with two, contiguous bases mismatched between targets and probes. In this case, no

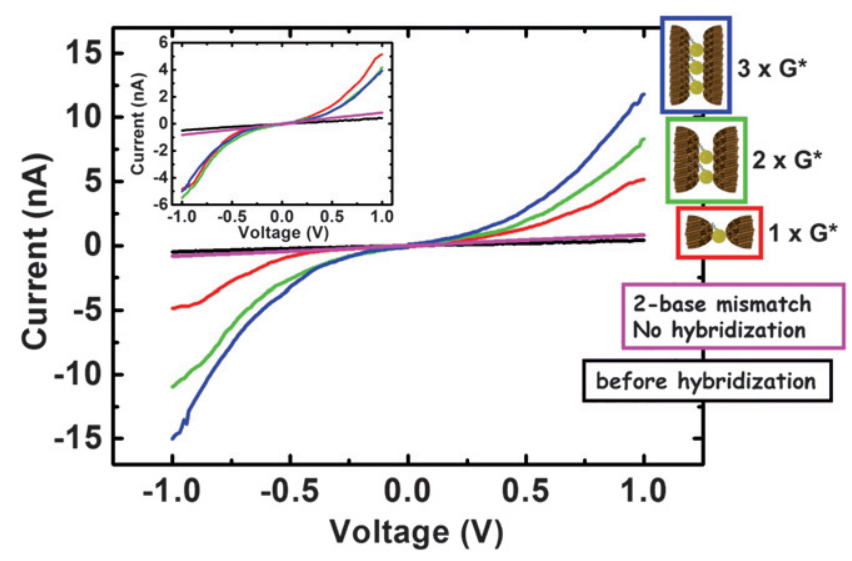

Fig. 2 Electrical detection. $I-V$ characteristics of various functionalized nanojunctions before hybridization (black curve), after hybridization with AuNC-DNA conjugates having ssDNA complementary to the capture probes on the electrodes (blue, green and red curves) and in the case of a two-base mismatch in the oligonucleotide sequences (magenta curve). In the case of the non-hybridized nanojunctions the current is negligible, whereas biorecognition events between complementary ssDNA can be detected as an increase in the current. The inset shows that the red, green, and blue curves collapse on almost the same characteristic if they are divided by the integer values 1,2 , and 3 . This leads us to conclude that one, two and three conjugates, respectively, bridge these nanojunctions resulting in conductance values at $1 \times, 2 \times$ and $3 \times G^{*}$. 
conduction variation was observed (magenta curve in Fig. 2) demonstrating the excellent selectivity of the device. ${ }^{7}$ These results are confirmed by the lack of AuNCs bridging the gap in SEM images of devices with the mismatched DNA sequences. When hybridization was performed with complementary DNA with no AuNCs, current was again in the hundreds of picoAmperes range.

In conclusion, we demonstrated for the first time a DNA-chip with electrical read-out and single-event sensitivity based on a new interconnection technique for cheap and massive fabrication of large-scale arrays of nanojunctions. Hybridization events are detected by the discrete increase in conductivity when the target AuNC-DNA conjugates bridge the electrode gap. By virtue of the very high sensitivity and ease of fabrication, this device is a building block for a new generation of highly sensitive, robust, low-cost nanosensors, which can be easily multiplexed with no need of amplification techniques (such as PCR). Compared to other electrical read-out schemes, ${ }^{8-11}$ our approach accomplishes two main improvements: (i) smaller size of the inter-electrode gap (a few nanometers) which makes single-event sensitivity possible and multiple layers of self-assembled gold nanoparticles for signal amplification between electrodes unnecessary; ${ }^{10,11}$ and (ii) no need of silver deposition for signal enhancement which increases the complexity of the detection process, and prevents the recycling of the chip which is here possible by increasing the temperature above the melting temperature. $^{8}$ Moreover, for proteomic applications, the thiol groups of cysteines available in the protein can be potentially employed for a strong bonding 5 and to avoid unfolding and loss of functionality. ${ }^{12}$

\section{Methods}

As conductive markers, we used AuNCs of $20 \mathrm{~nm}$ mean diameter purchased from Ted Pella, Inc. in aqueous solutions. Initially, their surface was passivated with citrate molecules which were then exchanged with bis( $p$-sulfonatophenyl)phenylphosphine (BSPP) in order to make them stable at micromolar concentrations and at the saline conditions needed for the hybridization reaction to occur. ${ }^{13}$ TEM images (not shown) confirm the absence of AuNC aggregates in solution.

The AuNC-DNA conjugates were prepared by addition of $5^{\prime}$-SH-ssDNA ${ }^{3}$ to the AuNC solution. As the accessibility of the oligonucleotides for hybridization depends on the conformation and packing of the ssDNA around the AuNCs, ${ }^{13}$ it is necessary to optimize the number, $N$, of oligonucleotides bound to a nanocrystal. ${ }^{14,15}$ Moreover, a precise control of $N$ (e.g. by electrophoretic isolation ${ }^{14}$ ) would allow quantitative information to be obtained on the number of hybridization events. Thus, the conjugates were analyzed by gel electrophoresis on $2 \%$ agarose gel after $18 \mathrm{~h}$ of reaction. Since the surface of phosphine-coated NCs is negatively charged like DNA, the conjugation changes the size but not the charge density, and thus the electrophoretic mobility will reflect the number of oligonucleotides attached per NC. ${ }^{13,14}$ As the number of strands attached to the AuNCs increases, the electrophoretic mobility of the conjugates compared to bare AuNCs is retarded, until a saturation mobility for molar ratios greater than 200 is achieved (see the ESI $\dagger$ ). No significant difference in the AuNC distribution was observed in
TEM images, suggesting a negligible presence of nanocrystal clusters mediated by non-specific DNA interaction. Since no difference was observed in gel of a 40:1 DNA:AuNC solution left to react $18 \mathrm{~h}$ or $42 \mathrm{~h}$, we conclude that no unreacted oligonucleotides remain in solution after $18 \mathrm{~h}$.

Both limiting cases with few or too many oligonucleotides per NC reduce the hybridization efficiency, respectively due to (1) a small number of reactive sites wrapped around the $\mathrm{Au}$ surface or (2) their excessive packing with problems of sterical hindrance. ${ }^{13}$ The ideal configuration is an intermediate case, ${ }^{13}$ where the inner part of the oligonucleotide is fully stretched, while the outer tail (in a random coil configuration) is highly accessible for efficient hybridization and also increases the colloidal stability at the salt concentration needed for the hybridization to occur. Since we found a saturation mobility (corresponding to a saturated AuNC surface) for DNA/AuNC molar ratios greater than 200, we used a DNA:AuNC molar ratio between 10:1 and 40:1.

To test the accessibility of oligonucleotides in conjugates and investigate the influence of the packing density, we probed the hybridization on a gold (on mica) surface functionalized with capture probes. We first realized an oriented SAM of $5^{\prime}-\mathrm{SH}-$ ssDNA. Compact SAMs obtained by $18 \mathrm{~h}$ of incubation at room temperature in a DNA solution $1 \mu \mathrm{M}$ in NaCl-TE buffer did not provide efficient hybridization sites due to the close packing of the capture probes which limits their accessibility. On the other hand, mixed SAMs of thiol oligonucleotides and (short) 2-mercaptoethanol molecules (which act as spacers) permit control over the packing density and, at the same time, reduce non-specific interactions between the individual oligonucleotides and the gold surface in a convenient way to favour hybridization. A mixed SAM has been realized by $2 \mathrm{~h}$ of incubation at room temperature in $500 \mu \mathrm{l}$ of a DNA solution ( $1 \mu \mathrm{M}$ DNA and $1 \mathrm{M} \mathrm{KH}_{2} \mathrm{PO}_{4}$ ) and $1 \mathrm{~h}$ of incubation in a mercaptoethanol solution (1 $\mathrm{mM}$ in water). Hybridization with DNA-AuNC conjugates was carried out at room temperature by incubating the functionalized surface for $6 \mathrm{~h}$ in $500 \mu \mathrm{l}$ of a 10:1 DNA/AuNC solution $0.25 \mathrm{nM}$ in PBS (phosphate buffer solution, $0.1 \mathrm{M} \mathrm{NaCl}$ ). Then samples were washed in PBS. In the case of mixed SAMs of capture probes and 2-mercaptoethanol, this procedure resulted in a homogeneous and compact layer of AuNCs (see the ESI $\dagger$ ), while only a few immobilized AuNCs were observed when compact SAMs of oligonucleotides were hybridized (not shown). The stringency of the binding events was demonstrated by a comparison with a sample, where another mixed SAM was hybridized with AuNCs conjugated to 5'-SH-ssDNA having a mismatch consisting of two, non-contiguous bases. No immobilized AuNCs were observed in this case (see the ESI $\dagger$ ).

\section{Acknowledgements}

The authors gratefully acknowledge the financial support by the European Union (EU-FP6-029002, acronym: SpiDME) and the Italian Institute of Technology (IIT). Moreover, we would like to thank Franco Calabi and Adriana Passaseo for fruitful discussions and providing materials, and Eliana D'Amone for technical assistance. 


\section{References}

1 M. C. Pirrung, Angew. Chem., Int. Ed., 2002, 41, 1276.

2 I. Amlani, A. M. Rawlett, L. A. Nagahara and R. K. Tsui, Appl. Phys. Lett., 2002, 80, 2761-2763.

3 The following sequences were used with a $10 \mathrm{nt}$-spacer after the thiol group at the $5^{\prime}$-terminus to facilitate hybridization: probe $5^{\prime}$-CTT TGG GGG ATG TGT TTT CCC TCA ACA CTT TGA TG-3' (on surfaces and electrodes); target $5^{\prime}$-TTC CAG TGG CCA TCA AAG TGT TGA GGG AAA ACA CA-3' (on AuNCs); modified (non-complementary) target $5^{\prime}$-TTC CAG TGG CCA TCA AAG TGC CGA GGG AAA ACA CA-3' (on AuNCs).

4 R. Krahne, A. Yacoby, H. Shtrikman, I. Bar-Joseph, T. Dadosh and J. Sperling, Appl. Phys. Lett., 2002, 81, 730-732.

5 G. Maruccio, P. Marzo, R. Krahne, A. Passaseo, R. Cingolani and R. Rinaldi, Small, 2007, 3, 1184-1188.

6 W. Wang, T. Lee and M. A. Reed, Phys. Rev. B: Condens. Matter Mater. Phys., 2003, 68, 035416.

7 The calculated melting temperature is around $54{ }^{\circ} \mathrm{C}$ for the complementary sequence and decreases to around $50{ }^{\circ} \mathrm{C}$ with the mismatch. Experiments were carried out at about $30^{\circ} \mathrm{C}$; however, the local temperature can be different due to the flowing of current through a very small area (a few nanometers). Moreover, it is well known that oligonucleotide-nanoparticle conjugates exhibit unique hybridization properties that can lead to improved selectivity in assays leading to very sharp melting transitions and were reported to allow differentiation of a perfectly complementary target strand from a strand with a single base mismatch on a 24-nucleotide sequence (Taton et al., Science, 2000, 289, 1757). On one side, this can be related to the presence of multiple strands on the $\mathrm{NC}$ and problems of sterical hindrance. Then, in the present case, the monitoring of a tunneling current is extremely sensitive to the particle-surface distance. This is another advantage of the proposed approach.

8 S. J. Park, T. A. Taton and C. A. Mirkin, Science, 2002, 295, 15031506.

9 Y. Fan, X. T. Chen, A. D. Trigg, C. H. Tung, J. M. Kong and Z. Q. Gao, J. Am. Chem. Soc., 2007, 129, 5437-5443.

10 Y. T. Cheng, C. C. Pun, C. Y. Tsai and P. H. Chen, Sens. Actuators, $B, 2005,109,249-255$.

11 C. Y. Tsai, Y. H. Tsai, C. C. Pun, B. Chan, T. Y. Luh, C. C. Chen, F. H. Ko, P. J. Chen and P. H. Chen, Microsyst. Technol., 2005, 11, 91-96.

12 G. Maruccio, A. Biasco, P. Visconti, A. Bramanti, P. P. Pompa, F. Calabi, R. Cingolani, R. Rinaldi, S. Corni, R. Di Felice, E. Molinari, M. R. Verbeet and G. W. Canters, Adv. Mater., 2005, 17, 816.

13 W. J. Parak, T. Pellegrino, C. M. Micheel, D. Gerion, S. C. Williams and A. P. Alivisatos, Nano Lett., 2003, 3, 33-36.

14 D. Zanchet, C. M. Micheel, W. J. Parak, D. Gerion and A. P. Alivisatos, Nano Lett., 2001, 1, 32-35.

15 T. Pellegrino, R. A. Sperling, A. P. Alivisatos and W. J. Parak, J. Biomed. Biotechnol., 2007, 26796. 